\title{
The Effects of Primary Unconjugated Bile Acids on Nanoencapsulated Pharmaceutical Formulation of Hydrophilic Drugs: Pharmacological Implications
}

\author{
Armin Mooranian $\mathbb{D}^{1,2}$ \\ Thomas Foster (D) ${ }^{1,2}$ \\ Corina M lonescu (iD) ${ }^{1,2}$ \\ Louise Carey $\mathbb{B}^{1,2}$ \\ Daniel Walker (D) ${ }^{1,2}$ \\ Melissa Jones ${ }^{1,2}$ \\ Susbin Raj Wagle (D) ${ }^{1,2}$ \\ Bozica Kovacevic ${ }^{1,2}$ \\ Jacqueline Chester (D) ${ }^{1,2}$ \\ Edan Johnstone ${ }^{1,2}$ \\ Jafri Kuthubutheen ${ }^{3}$ \\ Daniel Brown ${ }^{4}$ \\ Marcus D Atlas ${ }^{2}$ \\ Momir Mikov ${ }^{5}$ \\ Hani Al-Salami $\mathbb{D}^{1,2}$ \\ 'The Biotechnology and Drug \\ Development Research Laboratory, \\ Curtin Medical School \& Curtin Health \\ Innovation Research Institute, Curtin \\ University, Bentley, Perth, 6102, WA, \\ Australia; ${ }^{2}$ Hearing Therapeutics, Ear \\ Science Institute Australia, Queen \\ Elizabeth II Medical Centre, Nedlands, \\ Perth, 6009, WA, Australia; ${ }^{3}$ Fiona \\ Stanley Hospital, Perth, WA, Australia; \\ ${ }^{4}$ Curtin Medical School \& Curtin Health \\ Innovation Research Institute, Curtin \\ University, Perth, WA, Australia; \\ ${ }^{5}$ Department of Pharmacology, \\ Toxicology and Clinical Pharmacology, \\ Faculty of Medicine, University of Novi \\ Sad, Novi Sad, 21101 , Serbia
}

Correspondence: Hani Al-Salami

Hearing Therapeutics, Biotechnology and

Pharmaceutical Sciences, Curtin

University, Bentley, Perth, 6102, WA,

Australia

Tel +6I 892669816

$\mathrm{Fax}+61892662769$

Email hani.al-salami@curtin.edu.au
Introduction: In a recent study, in our laboratory, primary unconjugated bile acids, commonly found in humans, chenodeoxycholic acid (CDCA), have been shown to improve stability of nanoencapsulated lipophilic drugs and improve their release profile after oral administration likely via electrokinetic stabilisation. Hence, this study aimed to examine the effects of CDCA on exerting similar effects on hydrophilic drugs.

Methods: Various CDCA-based formulations were produced for the orally administered hydrophilic drug, metformin. Analyses of these formulations included electrokinetic potentials, topography, drug and CDCA formulation contents, nano size distribution, heat-induced deformation and outer-core expansion indices, release profiles, shell-resistance ratio, and thermal and chemical indices. With the drug's main target being pancreatic beta-cells, the formulations' effects on cell viability, functions and inflammatory profiles were also investigated.

Results and Conclusions: CDCA-based metformin formulations exhibited improved stability and release profiles via thermal, chemical and electrokinetic effects, which were formulation-dependent suggesting potential applications of CDCA in the oral targeted delivery of hydrophilic drugs.

Keywords: microencapsulation, diabetes mellitus, Glyceryl monooleate, Eudragit, chenodeoxycholic acid

\section{Introduction}

Bile acids are naturally produced in the human liver and play a key role in cholesterol metabolism and in maintaining bile acid homeostasis. ${ }^{1}$ In addition to this, bile acids are being increasingly recognised as complex integrators playing significant roles in immune function, signalling pathways and have been demonstrated to have an antidiabetic effect through signalling pathways. ${ }^{2}$ In addition to these direct physiological effects, linbile acids incorporated into micro and nano capsules have been shown to improve the stability and release profile of lipophilic drugs and improve targeted oral delivery likely due to electrokinetic stabilisation. ${ }^{3-5}$

CDCA is a primary bile acid that reduces cholesterol levels by increasing intestinal excretion and interfering with bile acid synthesis to reduce secretion of cholesterol and cholesterol saturation. ${ }^{6,7}$ It is commonly used to treat constipation and gall stones as well as lipid storage disorders, ${ }^{8}$ however, hepatotoxicity and gastrointestinal disorders have been shown to have adverse effects. ${ }^{6}$ Outside pharmacological applications recent studies have found that CDCA interacts with vitamin $\mathrm{D}$ receptors, with these playing a role in bile acid excretion. ${ }^{9}$ 
Lipophilic drugs have been encapsulated with CDCA, leading to improvements in stability. Mathavan et al incorporated CDCA into microcapsules containing the lipophilic drug gliclazide with an improvement in physical characteristics, stability, and release profiles. The change in zeta potential was hypothesised to be due to the surfactant properties of CDCA reducing charge on the outer surface of the molecules. ${ }^{10}$

Metformin is known as a biguanide drug and has been widely used for treating Type 2 diabetes. Despite its high efficacy profile, metformin uptake, particularly long-term, has been associated with multiple adverse events and exhibits a long list of side effects, including weight loss, diarrhoea and vomiting, which can be severe.

The incorporation of bile acids has been shown to modify surface charge, which is measured in the same manner as zeta potential, with subsequent benefits in microcapsule characteristics and drug release. Mooranian et al incorporated ursodeoxycholic acid (UDCA) into nano and microcapsules for encapsulation of the lipophilic drug probucol, with bile acid formulations showing enhanced electrokinetic stability and enhanced drug uptake. ${ }^{11}$ In another study, UDCA inclusion for $\beta$ cell encapsulation stabilised electrokinetic charge by enhancing electrostatic and free charge, improving the physical characteristics of the capsules and protective effects on encapsulated cells. ${ }^{12}$

The modification of electrokinetic potential has been shown to improve drug delivery systems and release across a range of pharmaceuticals. Bile acid incorporation with antibiotics has shown a shift in free charge and subsequent interaction between functional groups and micelles, allowing characterisation of the partitioning of solutes in micelles, as well as a way to optimise drug delivery systems, with a resultant increase in drug solubility. ${ }^{13}$ Gallardo et al demonstrated that stability is primarily controlled by zeta potential in polymeric drug delivery systems, with modification to a more favourable zeta potential yielding more homogenous suspensions less prone to flocculation. ${ }^{14}$ Ophthalmic application of aspirin was shown to be improved by encapsulating the drug with proteins that stabilised the electrokinetic potential, resulting in a sustained release of the drug with significantly reduced haemolysis. ${ }^{15}$

Eudragit (Eud) is a polymethacrylate-based polymer that, like BAs, acts as a useful excipient in the delivery of various pharmaceutical products. There are several different types of Eud polymers, each with different $\mathrm{pH}$-dependent and independent solubility. In general, Euds are classified based on charge (anionic, cationic or neutral). Cationic Eud is useful in taste modification, while non-ionic is useful in GIT targeting. ${ }^{16}$ Neutral Euds have previously been explored in capsule preparations of ketoprofen, resulting in delayed and $\mathrm{pH}$ independent drug release. ${ }^{17}$

Electrokinetic stabilisation improves the drug delivery systems, particularly the physical characteristics, stability, and release profiles of encapsulated drugs. Leveraging the physical and chemical stabilising properties of bile acids in encapsulation leads to an improvement in resistance to mechanical stress as well as improved surface characteristics and resistance to water permeation. The resultant modification of electrokinetic potential of bile acid incorporation and subsequent improvement in drug delivery is desirable. Additionally, the use of EU provides a novel way to further improve the release profile of drugs in microcapsules. This study aimed to investigate the impact of CDCA and Eud types (NM, RL, RS) incorporation in microcapsules to deliver the hydrophilic drug metformin (M), to establish if the same improvements seen in lipophilic drug delivery could be established.

\section{Materials and Methods \\ Materials}

Metformin (M, 98\%), sodium alginate (SA, 99\%) and chenodeoxycholic acid (CDCA, 99\%) purchased from Sigma Chemical Co, USA. Calcium chloride dihydrate $\left(\mathrm{CaCl}_{2} \cdot 2 \mathrm{H}_{2} \mathrm{O} 98 \%\right)$ was obtained from Scharlab S.L, Australia. All reagents and solvents were HPLC grade used without further purification or modification and were supplied by Merck (Australia).

\section{Drug Preparations}

Stock suspensions or pre-encapsulation material were prepared by adding $1.6 \mathrm{~g} \mathrm{SA}, 0.6 \mathrm{~g}$ of bile acid, 0.1 $\mathrm{g}$ Glyceryl monooleate, $3 \mathrm{~g}$ Poloxamer, $0.2 \mathrm{~g}$ of $\mathrm{M}$ powder and $1 \mathrm{~g}$ of Eud to $100 \mathrm{~mL}$ of ultrapure water and mixed by hand. The stock suspensions were then placed on a magnetic stirrer to mix thoroughly for 24 hours at room temperature before being stored in the refrigerator at $4^{\circ} \mathrm{C}$. Stock solutions were within 48 hours of preparation. A stock solution of $\mathrm{CaCl}_{2}(2 \%)$ was prepared by adding $2 \mathrm{~g} \mathrm{CaCl}_{2}$ powder to $100 \mathrm{~mL} \mathrm{HPLC}$ water and mixed thoroughly, until completely dissolved. 
Table I Formulation List for Preparation of Pre-Encapsulation Mixtures

\begin{tabular}{|l|l|}
\hline Formulation & Components \\
\hline FI & EudNM30D \\
F2 & EudNM30D + CDCA \\
F3 & EudRL30D \\
F4 & EudRL30D + CDCA \\
F5 & EudRS30D \\
F6 & EudRS30D + CDCA \\
\hline
\end{tabular}

\section{Microcapsule Preparation}

Capsules were prepared using a well-defined and heavily refined method that has been established by our laboratory. ${ }^{18-25}$ Pre-encapsulation material was prepared as with different classes of Eud added according to Table 1. Each formulation also contained $1.6 \%$ SA, $0.1 \%$ Glyceryl monooleate, $0.2 \% \mathrm{M}$ and $3 \%$ Poloxamer. Formulations F2, F4 and F6 had 0.6\% CDCA and each solution had $1 \%$ of the appropriate Eud as per Table 1 .

The Büchi encapsulator system was used to perform the Ionic Gelation Vibrational Jet Flow (IGVJF) encapsulation. ${ }^{26-30}$ Settings including vibration frequency, voltage and nozzle diameter were tuned to produce a uniform droplet size of pre-encapsulation material. Microcapsules were then cross-linked in a $2 \% \mathrm{CaCl}_{2}$ bath as consistently sized spheres. ${ }^{31-34}$ Capsules were then rinsed under deionised water for at least one minute prior to drying or any testing was to occur.

\section{Microcapsule Imaging}

To assess preliminary sizing and quantities capsules were imaged via light microscopy. Glass slides with a calibrated scale were loaded with freshly encapsulated material. 4X objective was then used with the Olympus IX-51 inverted microscope to assess the capsules..$^{19,35-37}$

Scanning electron microscopy (SEM) was also performed to review capsule topography. Freshly formed capsules were dried in a stability chamber for three days at $40^{\circ} \mathrm{C}$ and $40 \%$ humidity. Capsules were coated in $5 \mathrm{~nm}$ of platinum whilst under a vacuum to allow for imaging with the MIRA3 FibSEM SEM.

\section{Microcapsule Size, Electrokinetic Potential, and Stability}

In addition to microscopy, capsule size was determined by two further methods. Mie scattering and Fraunhofer diffraction is a rapid technique that utilises lasers to determine the size of particles with the use of lasers. ${ }^{38}$ The Mastersizer 2000 from Malvern Instruments was used to record the laser scatter from prepared particles to determine their size using Mie scattering and Fraunhofer diffraction. Sizing was also assessed through spectroscopy performed by the Zetasizer $3000 \mathrm{HSa}$ also from Malvern Instruments. Both instruments were run using standardised procedures already established by the laboratory. ${ }^{21}$ Electrokinetic potential aka zeta potential was also assessed using the Zetasizer $3000 \mathrm{HSa}$. Measurements were performed at $25^{\circ} \mathrm{C}$ and each sample was repeated in triplicate. ${ }^{18,21}$

Stability studies were carried out to evaluate the temperature resistance of the capsules. Pre-determined quantities of capsules were prepared and placed into sterile petri-dishes for analysis. Capsules were then stored at $-20^{\circ} \mathrm{C}, 5^{\circ} \mathrm{C}, 25^{\circ} \mathrm{C}$, and $40^{\circ} \mathrm{C}$. Humidity was set at $35 \%$, and capsules were stored for 3 days. Humidity and temperature were regulated to ensure consistent conditions. After the 3-day incubation, capsules were assessed for signs of breakdown.

\section{Characterisation of Loaded Capsules}

Drug loading and efficiency were determined via high performance liquid chromatography (HPLC) using an established method for determining M content. ${ }^{39-41}$ Briefly, $100 \mathrm{mg}$ of capsules were broken down and made up to $200 \mathrm{~mL}$ with phosphate buffer ( $\mathrm{pH}$ 7.4). Samples were centrifuged and the filtered for HPLC assessment. M concentration was then quantified by a UV detector set at a wavelength of $227 \mathrm{~nm}$ as per the laboratory methods. $\mathrm{M}$ loading was determined as a percentage of the weight of the $\mathrm{M}$ in the sample. Microencapsulation efficiency was determined as a percentage of the practical weight of $\mathrm{M}$ over the theoretical weight. Samples were repeated in triplicate.

\section{Mechanical Strength, Buoyancy, Chemical and Physical Compatibility}

Capsule expansion was assessed as a means to determine mechanical strength. Capsules were stored at $25^{\circ} \mathrm{C}$ and $37^{\circ} \mathrm{C}$ at $\mathrm{pH} 3$ and 7.4 to mimic the conditions inside the GIT. Capsule sizes were determined pre and post storage to determine a \% expansion.

Capsule buoyancy was also assessed. Buoyancy was calculated as the percentage of microcapsules that were able to float in $100 \mathrm{~mL}$ of phosphate buffered liquid. ${ }^{42}$

To determine physical and chemical compatibility, Fourier transform infrared (FTIR) and differential scanning calorimetric (DSC) analysis were performed as per 
our previous methods. ${ }^{19,37}$ FTIR assessed the absorption spectra of separate components, the combined mixtures and the drug containing capsules. Infrared assessment was performed on the PerkinElmer FTIR spectrometer TWO in the $650-4000 \mathrm{~cm}^{-1}$ scanning range, with results recorded at $25^{\circ} \mathrm{C}$. DSC was also performed on individual capsule components, mixtures, and crushed capsules on a DSC instrument (DSC 8000; PerkinElmer Inc., Waltham, MA, USA). $5 \mathrm{mg}$ of sample was prepared in aluminium vessels and heated in a nitrogen atmosphere at a rate of $20^{\circ} \mathrm{C} / \mathrm{min}$. Assays were repeated in triplicate.

\section{MTT and Inflammatory Biomarkers}

The MTT (3-[4,5-dimethylthiazol-2-yl]-2,5 diphenyl tetrazolium bromide) assay is a technique that was used to observe the effects of capsules on mitochondrial activity and by extension cell viability. ${ }^{43,44}$ Stock MTT solution was prepared at $5 \mathrm{mg} / \mathrm{mL}$ within 24 hours of use. All groups were treated in the exact same way, including controls. Cell concentrations per volume was set as $10^{6} \%$ $\mathrm{mL}$ and cells were randomly allocated to different groups.

Prepared MIN6 cells were added to each well of a 96 well plate and left to incubate for 24 hours, dried microcapsules were also stored in $\mathrm{pH} 7.4$ media for 24 hours at $37^{\circ} \mathrm{C}$ before being added to each well. ${ }^{45}$ Capsules with no cells were used as negative controls, plates were incubated for a further 24 hours. After the incubations, $20 \mu \mathrm{L}$ of the prepared MTT was added to each well. MTT was converted to MTT-formazan via mitochondrial reductase. MTT-formazan was then removed and dissolved in 100 $\mu \mathrm{L}$ dimethyl sulfoxide (DMSO) from Sigma Chemicals. The resultant purple solution was then analysed via photometry at 550nm. Findings are reported in triplicate.

MIN6 cells were also used to show inflammatory markers produced post exposure to the microcapsules. As above, capsules were incubated with cells for 48 hours and the media were then removed and analysed using the BD cytometric bead array (CBA). BD Mouse Flex Sets were used to detect tumour necrosis factor $\alpha$ (TNF- $\alpha$ ), interferon $\gamma$ (IFN- $\gamma$ ), interleukin-6 (IL-6), interleukin-10 (IL-10) and interleukin-1 (IL-1) via flow cytometry. Findings are reported in triplicate.

\section{Results and Discussion}

\section{Microscopy and Encapsulation Efficiency}

Microcapsule shape, size and topography appear to be consistent despite changes in formulation as seen in Figure 1. All capsules have consistently sized, oval, and 3D shapes as demonstrated in Figure 1A. Formulations F1, F2 and F5 appear to have smooth surface texture, while F4 and F6 have noticeable crystalline structures on their surface and F3 may have less obvious crystal-like structures. While no further analysis of the microcapsule surface was performed, it is possible that these are $\mathrm{M}$ on the surface of the capsule. ${ }^{46}$ This is consistent with other research by our laboratory that found anti-diabetic drug crystals on the surface of microcapsules. In these studies, these crystals were identified by X-ray spectroscopy and confirmed to be drugs. ${ }^{20,21,35}$ Other atoms including $\mathrm{Ca}$ and $\mathrm{Cl}$ have also been found on the surface of capsules and may result in the crystals formed
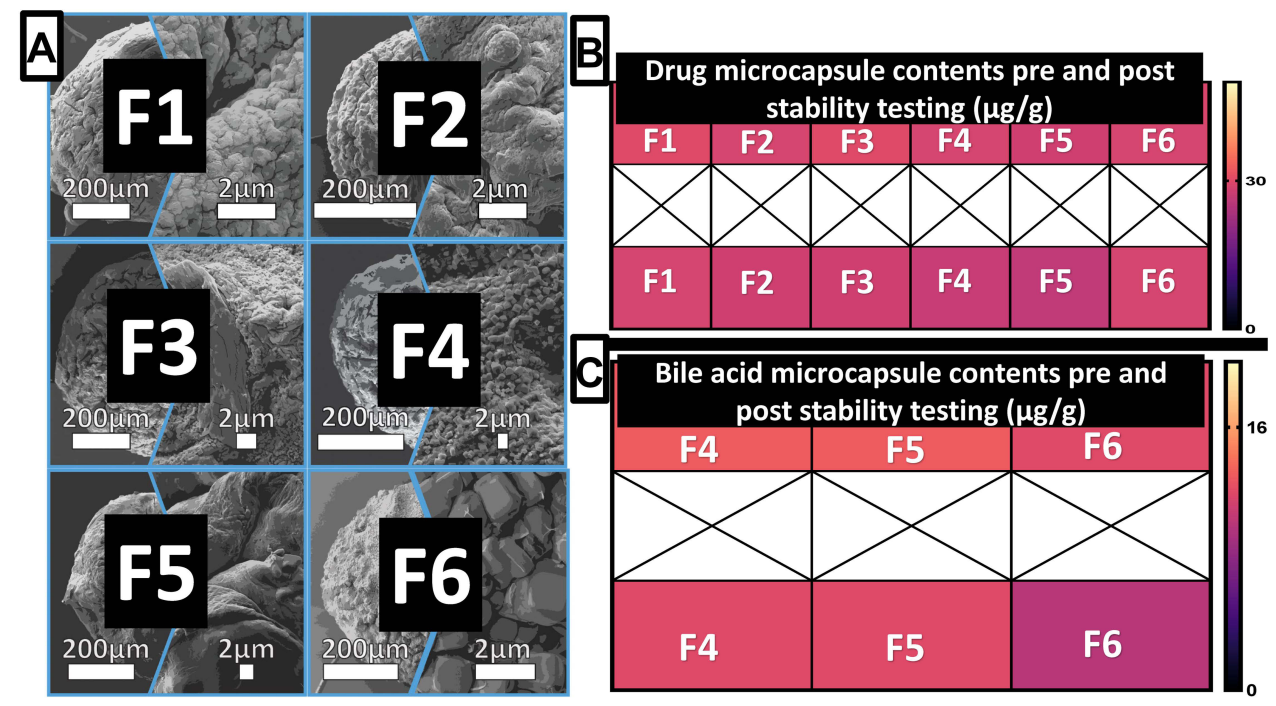

Figure I Microcapsule microscopy (A), Drug content pre and post encapsulation $(\mathbf{B})$ and Bile acid content pre and post encapsulation $(\mathbf{C})$. Data are mean \pm SEM. 
here. These crystals are commonly found in the ionic gelation processed microcapsules and are only seen due to the need to dry the capsules prior to imaging with the SEM. ${ }^{35}$

Drug content remained consistent across all formulations (F1-F6) when comparing pre and post-stability testing samples as seen in Figure 1B. Figure 1C shows bile acid content also remained consistent in capsules that contained CDCA. This suggests that neither drug nor bile acid is being broken down or significantly chemically modified by the encapsulation process. This is important as if they had been broken down, and the microcapsules may not be as effective as they could be. CDCA has been shown in other studies to not strongly interact with and therefore not potentially compromise drugs in microcapsules. ${ }^{10}$

\section{Microcapsule Size, Electrokinetic Potential, and Stability}

Figures $1 \mathrm{~A}$ and 2A show that all capsules (F1-F6) despite their composition maintained a consistent size and a mass of $\sim 800 \mu \mathrm{g}$. This indicates that the capsules were of similar size and mass, and therefore also of similar density despite differences in formulations.
Figure 2 also shows electrokinetic of the formulations. Figure 2B shows that F1 and F2 containing EudNM30D had a negative electrokinetic potential $\sim-20 \mathrm{mv}$. F3-F6 all had positive electrokinetic potential between $22-33 \mathrm{mv}$. CDCA did not appear to significantly change the potential in the capsules except for F3 and F4 (EudRL30D containing formulations) where there was a significant change in potential from 22 to $30 \mathrm{mv}(\mathrm{P}<0.05)$ with the addition of CDCA. In previous studies, CDCA has also been shown to increase the magnitude of the electrokinetic potential. ${ }^{18}$

Increased magnitude of electrokinetic potential (in either the positive or negative direction) results in increased repulsion between the molecules within the microcapsules. Increased repulsion is linked to increased stability and therefore strength. ${ }^{47}$ Increased electrokinetic potential is also useful in reducing particle aggregation, which is useful in improving drug delivery. A charge of $\sim 30 \mathrm{mv}$ is considered optimal for drug delivery. ${ }^{48}$

Heat-induced deformation is indicated in Figure 2C. Capsule formulations F1 and F2 showed breakdown at the lowest temperatures, beginning at $5^{\circ} \mathrm{C}$. CDCA marginally assisted in the stability by preventing early breakdown at

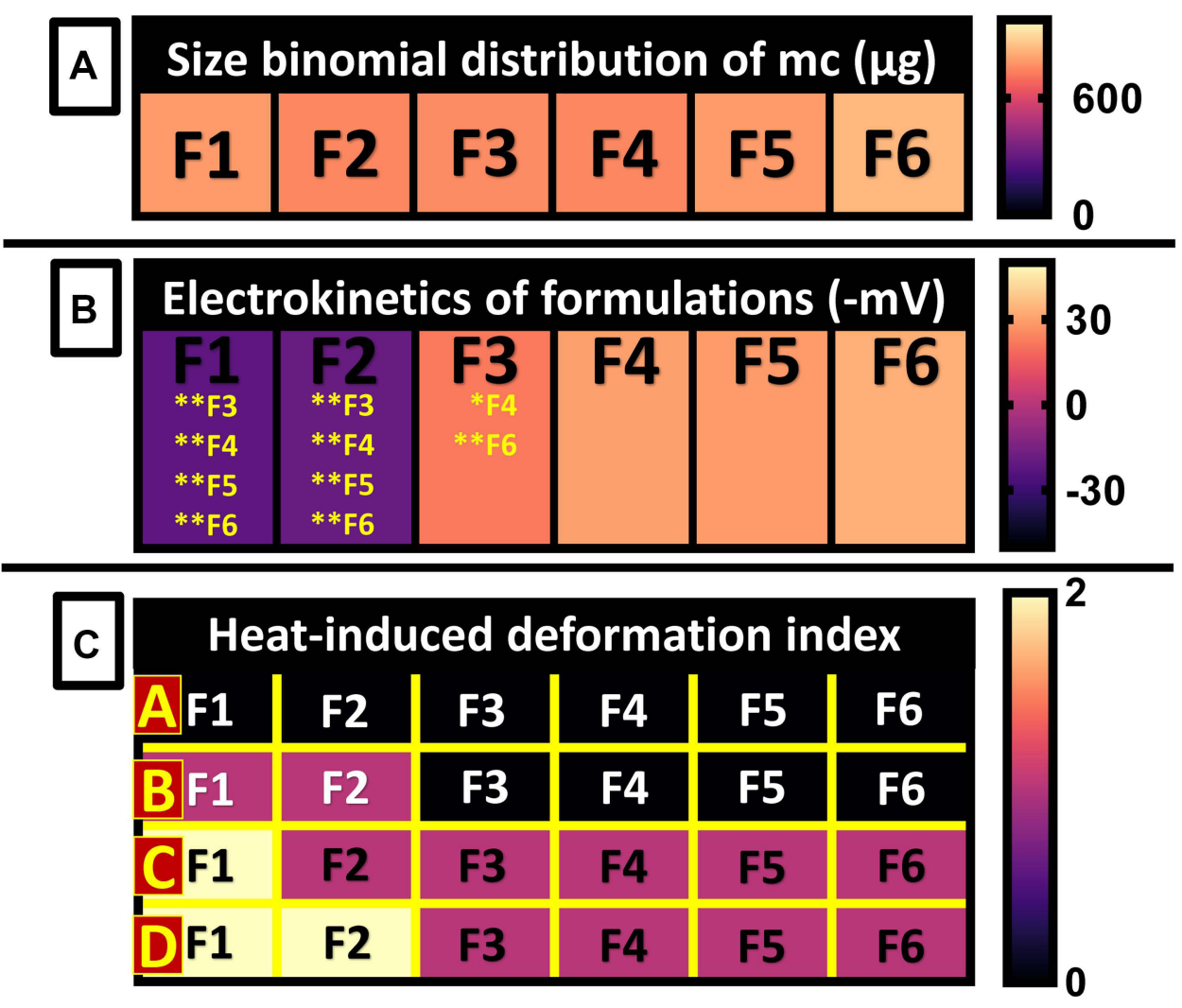

Figure 2 Microcapsule size distribution (A), electrokinetic (B) and stability (C). Stability was determined as heat-induced deformation index on a scale of $0-2$ at temperature A. $-20^{\circ} \mathrm{C}$ B. $5^{\circ} \mathrm{C} \mathrm{C.} 25^{\circ} \mathrm{C}$ and D. $40^{\circ} \mathrm{C}$. Data are mean $\pm \mathrm{SEM}, \mathrm{n}=3 . * \mathrm{P}<0.05, * * \mathrm{P}<0.01$. 
$25^{\circ} \mathrm{C}$ as demonstrated in $\mathrm{F} 2$ compared to $\mathrm{F} 1$. Formulations F3-F4 had consistent structural integrity, with no breakdown until $25^{\circ} \mathrm{C}$ and no further destruction at $40^{\circ} \mathrm{C}$. Optimally, breakdown would not occur until the capsules reach body temperature.

Given that the only difference between F1/3 and F3-4 was the use of a different Eud, it is likely that EudNM30D contributed to the early destruction of these capsules and its net negative electrokinetic potential. EudNM30D is considered a swellable polymer, and this may have contributed to its early breakdown. ${ }^{16}$

\section{Microcapsule Strength and Drug Release}

Capsule outer core expansion and drug release are shown in Figure 3. Outer core expansion is an indicator of osmosis across the microcapsule. Outer core expansion suggests that more water is getting into the capsules and expanding them. Generally, increased expansion indicates an increased risk of capsules breaking. Figure 3A-D shows capsule expansion remained consistent between capsules at different $\mathrm{pH}$. Previously, CDCA has been shown to improve capsule stability and increase capsule strength. Primarily CDCA was able to do this by reducing water permeation and thus decreasing swelling, increasing noncovalent bonding to improve strength and through deprotonation of carboxylic acid to improve electronegativity. ${ }^{18}$

Drug release profiles varied significantly based on the formulation as shown in Figure 3E. At $\mathrm{pH}$ 1.5, all formulations except $\mathrm{F} 1$ had consistent drug release. At $\mathrm{pH} 3.5$, formulation $\mathrm{F} 4$ released significantly more drugs than $\mathrm{F} 3$ $(\mathrm{P}<0.05)$ (CDCA negative) suggesting that CDCA presents is influencing drug release. At pH 6 F4, F5, and F6 released statistically significantly more drugs than F1, F2, and $\mathrm{F} 3$, this was also true at $\mathrm{pH} 7.4(\mathrm{P}<0.01)$. The differences between F3 and F4 indicate that in the EudRL30D containing formulation CDCA was influencing drug release. In other formulations, however, it does not appear that CDCA significantly affected drug release profiles. The $\mathrm{pH}$ dependent-release profiles seen in formulations F4-F6, where drug loading was achieved $>90 \%$ would be useful in the delivery of drugs through the GIT.

Previous work has indicated that the introduction of bile acids does improve the release profiles of drugs. ${ }^{4,5,49-53}$ Bile acids have been shown to effectively control drug release profiles. The bile acid deoxycholic acid has been shown at low concentrations to increase drug release from microcapsules while at higher concentrations decrease drug release. ${ }^{54}$ Eud formulations have been found to be effective in vivo at tolerating $\mathrm{pH}$ changes that mimic the colon and improving drug delivery. ${ }^{55}$ The introduction of Eud into bile acid containing capsules has also been shown to improve release profiles of drugs. ${ }^{56}$

\section{Shell Resistance, Gut-Sinkability, Chemical and Thermal Indexes}

Buoyancy (gut-sinkability) was consistent between microcapsules regardless of excipient content as seen in Figure 4B. This indicates a similar density between the capsules. It is important that buoyancy remains low, so that capsules will be able to retain significant contact with the surface of the gut rather than floating on top. This gives capsules the highest chance of interacting with the epithelial cells to release their contents.

Shell resistance in Figure 4A also remained consistent between capsules regardless of formulations. Suggesting that Eud type and CDCA did not affect shell resistance.

DSC and FTIR results in Figure 3C-F indicate no chemical modification to metformin. This indicates that the encapsulation process did not significantly alter the chemical structure of metformin, which implies that it would remain biologically active. This is in line with our previous work that has shown little compromise in the chemical structure of drugs or excipients during the encapsulation process (Figure 4). ${ }^{19,37,57}$

\section{Cell Survival and Inflammatory Biomarkers}

Cell survival is indicated in Figure 5A. Overall, cell survival studies were poor with F1-F6 all showing decreased survival compared with untreated cells $(\mathrm{P}<0.01)$ except for F4 which showed no significant change in cell survival. CDCA did not significantly modify the cell survival in sets F1/F2 or F5/F6. CDCA did, however, statistically significantly improve the survival of CDCA-EudRL30D containing capsule F4 compared to F3. In line with the findings in F4, previous studies have indicated that CDCA improved survival of cells that are encapsulated by CDCA. ${ }^{18}$ In previous studies CDCA has been found to be toxic in rhesus monkey liver, this was, however, only indicated in significantly higher doses than seen here. ${ }^{58}$ The lack of decreased survival is still positive as it indicates no detrimental effects to cells.

Figure 5B displays data about the insulin production of cells treated with capsules. All capsule treated cells produced insulin, while untreated cells did not. While 

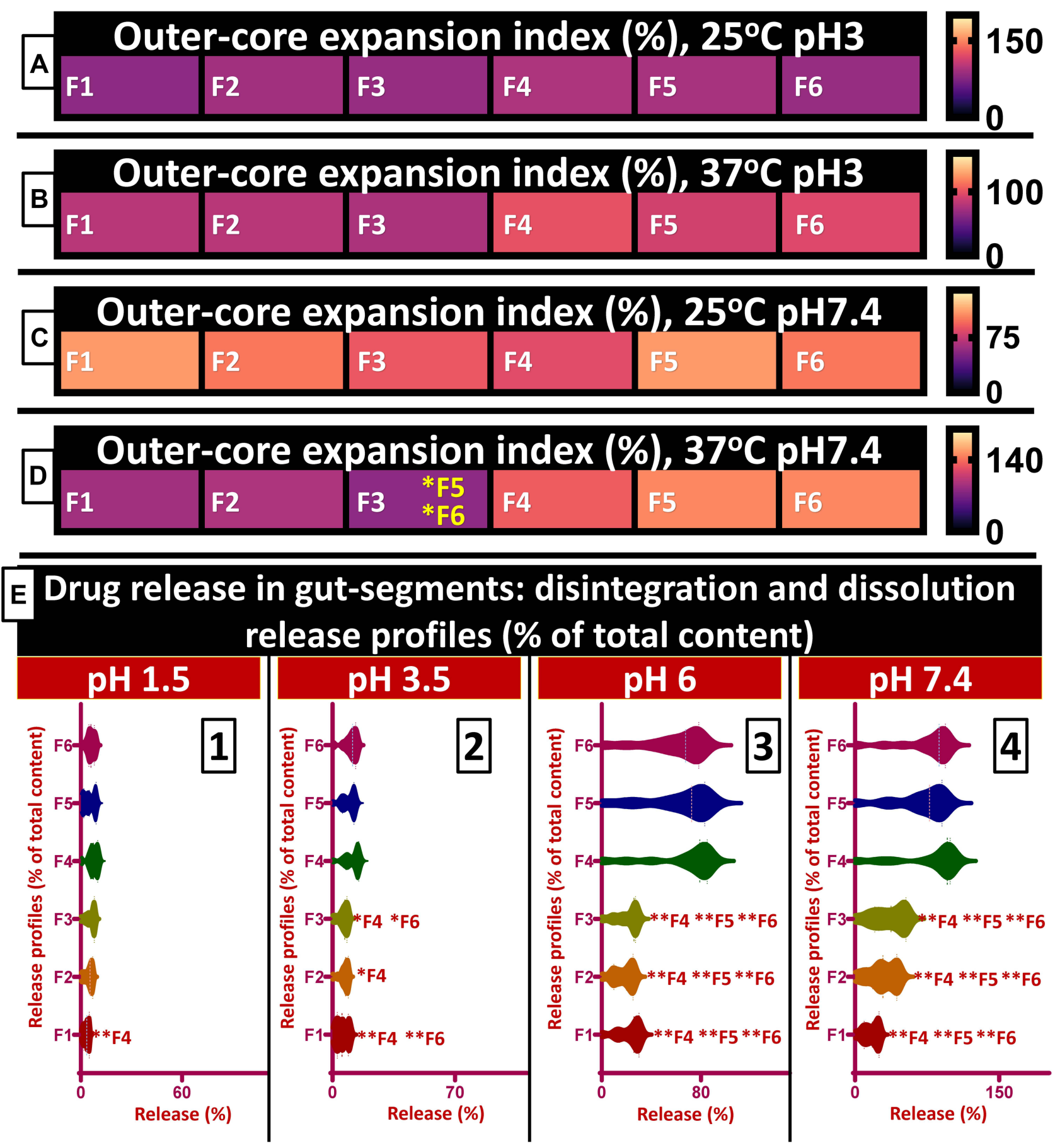

Figure 3 Microcapsule expansion (A-D) and drug release profiles $(\mathbf{E})$ at $\mathrm{pH}$ I.5 [E-1], $\mathrm{pH} 3.5[\mathrm{E}-2], \mathrm{pH} 6[\mathrm{E}-3]$ and $\mathrm{pH} 7.4[\mathrm{E}-4]$. Data are mean $\pm \mathrm{SEM}, \mathrm{n}=3 .{ }^{*}<0.05$, $* * \mathrm{P}<0.01$.

CDCA containing formulation F4 did result in the highest insulin production, it was not a statistically significant increased suggesting that CDCA does not influence insulin production of cells. This is in opposition to research that has found the stimulation of the Bile acid Farnesoid X receptor (FXR) has been shown to increase the production and secretion of insulin from cells. ${ }^{59}$ Diabetic animal models treated with Eud-Bile acid capsules have previously also shown no change in insulin concentration, and despite this, they did show improved control glycaemia. ${ }^{60}$ It is unclear why CDCA alone is capable of modifying insulin release, but with Eud, it is not able to significantly influence its release. 


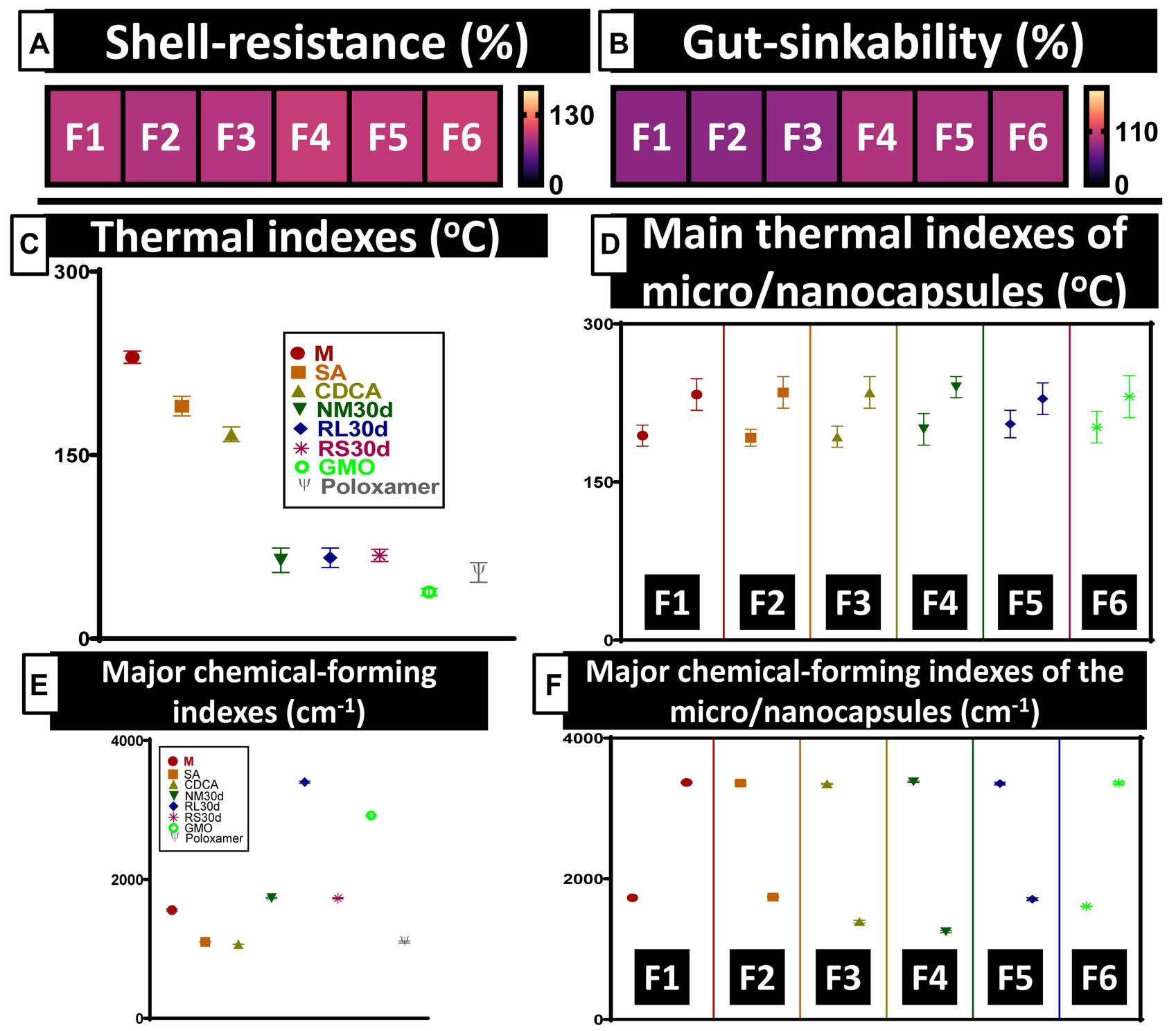

Figure 4 Shell resistance (\%) (A), gut sinkability (\%) (B, major thermal indexes of microcapsules (C and $\mathbf{D})$ and major chemical-forming indexes of microcapsules (E and $\mathbf{F})$.

Figure 5C-G shows inflammation markers produced by cells. Compared to untreated cells, all treated cells did not show statistically significant changes in TNF- $\alpha$, IFN- $\gamma$ or IL1 (Figure 5C, D, and F). Although not statistically significant, F4 did have the lowest levels of TNF- $\alpha$ and IFN- $\gamma$ as well as the second lowest level of IL-1. IL-6 was increased in all treated cells (Figure 5E); however, it was the least significantly increased in formulation $F 1, F 2$ and $F 4$ $(\mathrm{P}<0.05)$. IL-10 was only statistically significantly decreased in formulation $\mathrm{F} 4(\mathrm{P}<0.05)$ as seen in Figure 5G.

In vivo, CDCA is thought to trigger inflammation in liver fibrosis through stimulated secretion of inflammatory cytokines including TNF- $\alpha$, IL-1 and IL-6 through NLRP3 activation. ${ }^{61}$ Previous studies, however, have indicated that CDCA treatment of cells results in decreased TNF- $\alpha$ production. ${ }^{18}$ High levels of TNF- $\alpha$ have been shown to significantly impair cells' ability to survive. ${ }^{62}$ Other Eudbased formulations have also previously shown a decrease in TNF- $\alpha$ production. ${ }^{63}$

IFN- $\gamma$ alone is not capable of inducing apoptosis in pancreatic cells. This is in combination with TNF- $\alpha$ that it is able to induce apoptosis through STAT1 activation. ${ }^{64}$ Previous studies have replicated the findings that Eud-Bile acid-based formulations do not significantly alter the levels of IFN $-\gamma^{42}$ Interestingly, other bile acids have been shown to increase IFN- $\gamma$ levels. ${ }^{65}$

FXR activation by bile acids, including CDCA, has been shown to stimulate levels of IL- 6 as seen in this 


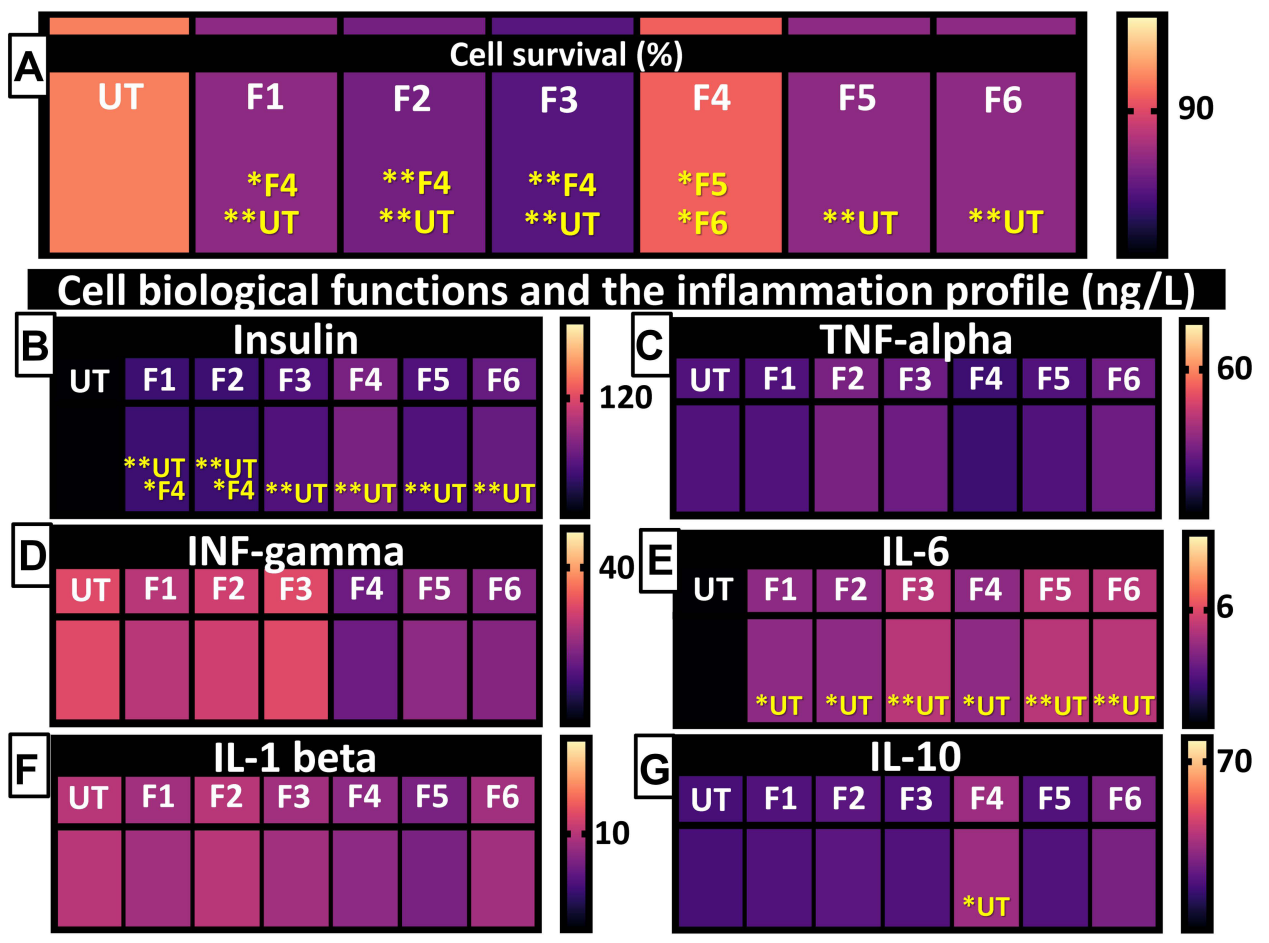

Figure 5 Cell survival (A), insulin production $(\mathbf{B})$, inflammatory markers $(\mathbf{C}-\mathbf{G})$. Data are mean $\pm \mathrm{SEM}, \mathrm{n}=3 .{ }^{*} \mathrm{P}<0.05, * * \mathrm{P}<0.0 \mathrm{I}$. Abbreviation: UT, untreated.

study. ${ }^{66}$ Previous work by our laboratory has, however, indicated that CDCA decreased IL-6 as well as IL-1 expression in treated cells. ${ }^{18,67-70}$

\section{Conclusion}

CDCA-Eud-based metformin formulations exhibited improved stability and release profiles via thermal, chemical, and electrokinetic effects, which were formulationdependent suggesting potential applications of CDCA in the oral targeted delivery of hydrophilic drugs.

Formulation F4, containing EudRL30D + CDCA showed the most potential. F4 resulted in smooth surface morphology with sizing consistent with other capsules. Drug and bile acid content did not have any statistically significant change between pre and post testing. F4 also showed an increased magnitude of electrokinetic potential with good shell resistance and gut-sinkability. F4 also showed cell survival on par with untreated cells but with increased insulin production and decreased inflammatory cytokine production. F4 also resulted in statistically significant improvement compared to F3 (EudRL30D, no CDCA) suggesting that bile acid content contributed to its improved parameters. Hence, the findings demonstrated advantages of CDCA-based metformin formulations in terms of better stability and release profiles which may result in improved safety profiles and potentially metformin efficacy. Further investigations into Eud-CDCA-based microcapsules are required to gain more insight into their effectiveness as drug excipients. F4 in particular would benefit from further investigation.

\section{Acknowledgments}

The authors acknowledge the Australian Postgraduate Award and the Curtin Research Scholarship for the support. The authors acknowledge the use of laboratory equipment, and the scientific and technical assistance of the Curtin University Electron Microscope Facility, which has been partially funded by the University, State and Commonwealth Governments. MIN-6 cells were provided by the University of Western Australia and their acquirement and use was approved by Curtin University's institutional guidelines.

\section{Funding}

$\mathrm{H}$ Al-Salami has been and is currently receiving funding from Beijing Nat-Med Biotechnology Co. Ltd. The work is partially supported by the European Union Horizon 2020 research project and innovation program under the Marie Skłodowska-Curie Grant Agreement No 872370. 
Curtin Faculty ORS-WAHAI Consortium and the Australian National Health and Medical Research (APP9000597).

\section{Disclosure}

Hani Al-Salami reports grants from Beijing Nat-Med Biotechnology Co. Ltd, European Union Horizon 2020 research project, Curtin Faculty ORS-WAHAI Consortium, and Australian National Health and Medical Research, during the conduct of the study. Jafri Kuthubutheen, Hani AlSalami, Armin Mooranian, and Daniel Brown report a patent Australian Provisional Patent Application No. 2020901933 "Treatment methods and formulations". Hani Al-Salami and Armin Mooranian report a patent Encapsulation Technology in cannabinoids oral delivery. The authors report no other potential conflicts of interest for this work.

\section{References}

1. Monte MJ, Marin JJ, Antelo A, Vazquez-Tato J. Bile acids: chemistry, physiology, and pathophysiology. World J Gastroenterol. 2009;15 (7):804-816. doi:10.3748/wjg.15.804

2. Thomas C, Pellicciari R, Pruzanski M, Auwerx J, Schoonjans K. Targeting bile-acid signalling for metabolic diseases. Nat Rev Drug Discov. 2008;7(8):678-693. doi:10.1038/nrd2619

3. Mooranian A, Raj Wagle S, Kovacevic B, et al. Bile acid bio-nanoencapsulation improved drug targeted-delivery and pharmacological effects via cellular flux: 6-months diabetes preclinical study. Sci Rep. 2020;10(1):1-15. doi:10.1038/s41598-019-53999-1

4. Wagle SR, Walker D, Kovacevic B, et al. Micro-nano formulation of bile-gut delivery: rheological, stability and cell survival, basal and maximum respiration studies. Sci Rep. 2020;10(1):1-10. doi:10.1038/ s41598-020-64355-z

5. Mooranian A, Zamani N, Takechi R, et al. Pharmacological effects of nanoencapsulation of human-based dosing of probucol on ratio of secondary to primary bile acids in gut, during induction and progression of type 1 diabetes. Artif Cells Nanomed Biotechnol. 2018;46 (sup3):S748-S754. doi:10.1080/21691401.2018.1511572

6. Khairy MA, Mansour FR. Simultaneous determination of ursodeoxycholic acid and chenodeoxycholic acid in pharmaceutical dosage form by HPLC-UV detection. J AOAC Int. 2017;100(1):59-64. doi:10.5740/jaoacint.16-0176

7. Ghosh Laskar M, Eriksson M, Rudling M, Angelin B. Treatment with the natural FXR agonist chenodeoxycholic acid reduces clearance of plasma LDL whilst decreasing circulating PCSK9, lipoprotein(a) and apolipoprotein C-III. J Intern Med. 2017;281(6):575-585. doi:10. 1111/joim. 12594

8. Sundaram SS, Bove KE, Lovell MA, Sokol RJ. Mechanisms of disease: inborn errors of bile acid synthesis. Nat Clin Pract Gastroenterol Hepatol. 2008;5(8):456-468. doi:10.1038/ncpgasthep1179

9. Nishida S, Ishizawa M, Kato S, Makishima M. Vitamin D receptor deletion changes bile acid composition in mice orally administered chenodeoxycholic acid. J Nutr Sci Vitaminol. 2020;66(4):370-374. doi:10.3177/jnsv.66.370

10. Mathavan S, Chen-Tan N, Arfuso F, Al-Salami H. The role of the bile acid chenodeoxycholic acid in the targeted oral delivery of the anti-diabetic drug gliclazide, and its applications in type 1 diabetes. Artif Cells Nanomed Biotechnol. 2016;44(6):1508-1519. doi:10.310 9/21691401.2015.1058807
11. Mooranian A, Zamani N, Mikov M, et al. Bio micro-nano technologies of antioxidants optimised their pharmacological and cellular effects, ex vivo, in pancreatic $\beta$-cells. Nanotechnol Sci Appl. 2020;13:1-9. doi:10.2147/NSA.S212323

12. Mooranian A, Negrulj R, Takechi R, Jamieson E, Morahan G, AlSalami H. Electrokinetic potential-stabilization by bile acid-microencapsulating formulation of pancreatic beta-cells cultured in high ratio poly-L-ornithine-gel hydrogel colloidal dispersion: applications in cell-biomaterials, tissue engineering and biotechnological applications. Artif Cells Nanomed Biotechnol. 2018;46(6):1156-1162.

13. Mrestani Y, Marestani Z, Neubert RH. The effect of a functional group in penicillin derivatives on the interaction with bile salt micelles studied by micellar electrokinetic chromatography. Electrophoresis. 2001;22(16):3573-3577. doi:10.1002/1522-2683(200109)22:16<3573::AID-ELPS3573>3.0.CO;2-K

14. Gallardo V, Morales ME, Ruiz MA, Delgado AV. An experimental investigation of the stability of ethylcellulose latex: correlation between zeta potential and sedimentation. Eur J Pharm Sci. 2005;26(2):170-175. doi:10.1016/j.ejps.2005.05.008

15. Das S, Bellare JR, Banerjee R. Protein based nanoparticles as platforms for aspirin delivery for ophthalmologic applications. Colloids Surf B Biointerfaces. 2012;93:161-168. doi:10.1016/j.colsurfb.2011.12.033

16. Thakral S, Thakral NK, Majumdar DK. Eudragit ${ }^{\circledR}$ : a technology evaluation. Expert Opin Drug Deliv. 2013;10(1):131-149. doi:10. $1517 / 17425247.2013 .736962$

17. Goto S, Kawata M, Nakamura M, Maekawa K, Aoyama T. Eudragit $\mathrm{RS}$ and $\mathrm{RL}$ (acrylic resins) microcapsules as $\mathrm{pH}$ insensitive and sustained release preparations of ketoprofen. J Microencapsul. 1986;3(4):293-304. doi:10.3109/02652048609021799

18. Mooranian A, Negrulj R, Al-Salami H. Primary bile acid chenodeoxycholic acid-based microcapsules to examine $\beta$-cell survival and the inflammatory response. BioNanoScience. 2016;6:103-109. doi:10.1007/s12668-016-0198-9

19. Mooranian A, Negrulj R, Chen-Tan N, et al. Microencapsulation as a novel delivery method for the potential antidiabetic drug, Probucol. Drug Des Devel Ther. 2014;8:1221.

20. Mooranian A, Negrulj R, Chen-Tan N, Watts GF, Arfuso F, AlSalami H. An optimized probucol microencapsulated formulation integrating a secondary bile acid (deoxycholic acid) as a permeation enhancer. Drug Des Devel Ther. 2014;8:1673.

21. Mooranian A, Negrulj R, Mikov M, Golocorbin-Kon S, Arfuso F, AlSalami H. Novel chenodeoxycholic acid-sodium alginate matrix in the microencapsulation of the potential antidiabetic drug, probucol. An in vitro study. J Microencapsul. 2015;32:589-597. doi:10.3109/ 02652048.2015.1065922

22. Mooranian A, Negrulj R, Al-Salami H. The influence of stabilized deconjugated ursodeoxycholic acid on polymer-hydrogel system of transplantable NIT-1 cells. Pharm Res. 2016;33(5):1182-1190. doi:10.1007/s11095-016-1863-y

23. Mooranian A, Negrulj R, Al-Sallami H, et al. Probucol release from novel multicompartmental microcapsules for the oral targeted delivery in type 2 diabetes. AAPS PharmSciTech. 2015;16(1):45-52. doi:10.1208/s12249-014-0205-9

24. Mooranian A, Negrulj R, Mathavan S, et al. Stability and release kinetics of an advanced gliclazide-cholic acid formulation: the use of artificial-cell microencapsulation in slow release targeted oral delivery of antidiabetics. J Pharm Innov. 2014;9:150-157. doi:10.1007/ s12247-014-9182-5

25. Negrulj R, Mooranian A, Al-Salami H. Potentials and limitations of bile acids in type 2 diabetes mellitus: applications of microencapsulation as a novel oral delivery system. J Endocrinol Diabetes Mellit. 2013;1:49-59.

26. Mathavan S, Ionescu CM, Kovacevic B, et al. Formulation buoyancy of nanoencapsulated gliclazide using primary, conjugated and deconjugated bile acids. Ther Deliv. 2019;10(9):573-583. doi:10.4155/tde-2019-0058 
27. Mamo JCL, Lam V, Brook E, et al. Probucol prevents blood-brain barrier dysfunction and cognitive decline in mice maintained on prodiabetic diet. Diab Vasc Dis Res. 2019;16(1):87-97. doi:10.1177/ 1479164118795274

28. Mooranian A, Negrulj R, Takechi R, et al. Alginate-combined cholic acid increased insulin secretion of microencapsulated mouse cloned pancreatic $\beta$ cells. Ther Deliv. 2017;8(10):833-842. doi:10.4155/tde2017-0042

29. Mooranian N, Al-Salami H. The effects of ionic gelation- vibrational jet flow technique in fabrication of microcapsules incorporating $\beta$ cell: applications in type-1 diabetes. Curr Diabetes Rev. 2017;13 (1):91-96. doi:10.2174/1573399812666151229101756

30. Negrulj R, Mooranian A, Chen-Tan N, et al. Swelling, mechanical strength, and release properties of probucol microcapsules with and without a bile acid, and their potential oral delivery in diabetes. Artif Cells Nanomed Biotechnol. 2016;44(5):1290-1297. doi:10.3109/ 21691401.2015.1024845

31. Mooranian A, Zamani N, Takechi R, et al. Modulatory nano/micro effects of diabetes development on pharmacology of primary and secondary bile acids concentrations. Curr Diabetes Rev. 2020;16 (8):900-909. doi:10.2174/1389450121666200204115121

32. Mooranian A, Zamani N, Mikov M, et al. A second-generation micro/ nano capsules of an endogenous primary un-metabolised bile acid, stabilized by Eudragit-alginate complex with antioxidant compounds. Saudi Pharm J. 2020;28(2):165-171. doi:10.1016/j.jsps.2019.11.017

33. Mooranian A, Zamani N, Mikov M, et al. Bio micro-nano technologies of antioxidants optimised their pharmacological and cellular effects, ex vivo, in pancreatic $\beta$-cells. Nanotechnol Sci Appl. 2020;13:1-9.

34. Mooranian A, Zamani N, Kovacevic B, et al. Pharmacological effects of secondary bile acid microparticles in diabetic murine model. Curr Diabetes Rev. 2020. doi:10.2174/1573399816666200626213735.

35. Mooranian A, Negrulj R, Al-Salami H. The incorporation of watersoluble gel matrix into bile acid-based microcapsules for the delivery of viable $\beta$-cells of the pancreas, in diabetes treatment: biocompatibility and functionality studies. Drug Deliv Transl Res. 2016;6 (1):17-23. doi:10.1007/s13346-015-0268-5

36. Mooranian A, Negrulj R, Arfuso F, Al-Salami H. Characterization of a novel bile acid-based delivery platform for microencapsulated pancreatic $\beta$-cells. Artif Cells Nanomed Biotechnol. 2016;44: 194-200. doi:10.3109/21691401.2014.934457

37. Mooranian A, Negrulj R, Arfuso F, Al-Salami H. Multicompartmental, multilayered probucol microcapsules for diabetes mellitus: formulation characterization and effects on production of insulin and inflammation in a pancreatic $\beta$-cell line. Artif Cells, Nanomed Biotechnol. 2016;44 (7):1642-1653. doi:10.3109/21691401.2015.1069299

38. de Boer GB, de Weerd C, Thoenes D, Goossens HW. Laser diffraction spectrometry: Fraunhofer diffraction versus Mie scattering. Part Part Syst Charact. 1987;4(1-4):14-19. doi:10.1002/ppsc.19870040104

39. Gedawy A, Al-Salami H, Dass CR. Development and validation of a new analytical HPLC method for simultaneous determination of the antidiabetic drugs, metformin and gliclazide. J Food Drug Anal. 2019;27(1):315-322. doi:10.1016/j.jfda.2018.06.007

40. Gedawy A, Dass CR, Al-Salami H. Polydimethylsiloxane-customized nanoplatform for delivery of antidiabetic drugs. Ther Deliv. 2020;11(7):415-429. doi:10.4155/tde-2020-0049

41. Gedawy A, Al-Salami H, Dass CR. Advanced and multifaceted stability profiling of the first-line antidiabetic drugs metformin, gliclazide and glipizide under various controlled stress conditions. Saudi Pharm J. 2020;28(3):362-368. doi:10.1016/j.jsps.2020.01.017

42. Mooranian A, Zamani N, Mikov M, et al. Novel nano-encapsulation of probucol in microgels: scanning electron micrograph characterizations, buoyancy profiling, and antioxidant assay analyses. Artif Cells, Nanomed Biotechnol. 2018;46:S741-S7. doi:10.1080/21691401.2018.1511571

43. Van Meerloo J, Kaspers GJ, Cloos J. Cell sensitivity assays: the MTT assay.Methods Mol Biol. 2011;731:237-245. doi:10.1007/978-161779-080-5_20.
44. Mooranian A, Negrulj R, Chen-Tan N, et al. Advanced bile acidbased multi-compartmental microencapsulated pancreatic $\beta$-cells integrating a polyelectrolyte-bile acid formulation, for diabetes treatment. Artif Cells Nanomed Biotechnol. 2016;44(2):588-595. doi:10.3109/21691401.2014.971806

45. Nakashima K, Kanda Y, Hirokawa Y, Kawasaki F, Matsuki M, Kaku K. MIN6 is not a pure beta cell line but a mixed cell line with other pancreatic endocrine hormones. Endocr J. 2009;56:45-53.

46. Patel KD, Patel NK. Formulation and evaluation of metformin hydrochloride microparticles by emulsion solvent evaporation technique. J Drug Deliv Ther. 2013;3(2). doi:10.22270/jddt.v3i2.471

47. Vinothini K, Rajan M. Chapter 9 - Mechanism for the nano-based drug delivery system. In: Mohapatra SS, Ranjan S, Dasgupta N, Mishra RK, Thomas S, editors. Characterization and Biology of Nanomaterials for Drug Delivery. Elsevier; 2019:219-263. doi:10.1016/B978-0-12-814031-4.00009-X

48. Gumustas M, Sengel-Turk CT, Gumustas A, Ozkan SA, Uslu B. Chapter 5 - Effect of polymer-based nanoparticles on the assay of antimicrobial drug delivery systems. In: Grumezescu AM, editor. Multifunctional Systems for Combined Delivery, Biosensing and Diagnostics. Elsevier; 2017:67-108.

49. Wagle SR, Kovacevic B, Walker D, et al. Alginate-based drug oral targeting using bio-micro/nano encapsulation technologies. Expert Opin Drug Deliv. 2020;17(10):1361-1376. doi:10.1080/17425247.2020.1789587

50. Kecman S, Škrbić R, Badnjevic Cengic A, et al. Potentials of human bile acids and their salts in pharmaceutical nano delivery and formulations adjuvants. Technol Health Care. 2020;28(3):325-335. doi:10.3233/THC-191845

51. Mooranian A, Zamani N, Mikov M, et al. Novel nano-encapsulation of probucol in microgels: scanning electron micrograph characterizations, buoyancy profiling, and antioxidant assay analyses. Artif Cells Nanomed Biotechnol. 2018;46(sup3):S741-S747.

52. Mooranian A, Zamani N, Mikov M, et al. Eudragit ${ }^{\circledR}$-based microcapsules of probucol with a gut-bacterial processed secondary bile acid. Ther Deliv. 2018;9(11):811-821. doi:10.4155/tde-2018-0036

53. Mooranian A, Negrulj R, Takechi R, Mamo J, Al-Sallami H, AlSalami H. The biological effects of the hypolipidaemic drug probucol microcapsules fed daily for 4 weeks, to an insulin-resistant mouse model: potential hypoglycaemic and anti-inflammatory effects. Drug Deliv Transl Res. 2018;8(3):543-551. doi:10.1007/s13346-017-0473-5

54. Tan Q, Bie M, Wang Z, et al. Insights into the mechanism of bile salt aggregates forming in a PEGylated amphiphilic polymer/bile salt mixed micelle. ChemistrySelect. 2018;3(13):3641-3651. doi:10. 1002/slct.201800382

55. Kshirsagar S, Bhalekar M, Umap R. In vitro in vivo comparison of two $\mathrm{pH}$ sensitive Eudragit polymers for colon specific drug delivery. J Pharm Sci Res. 2009;1(4):61.

56. Mooranian A, Zamani N, Mikov M, et al. Eudragit ${ }^{\circledR}$-based microcapsules of probucol with a gut-bacterial processed secondary bile acid. Ther Deliv. 2018;9(11):811-821.

57. Mooranian A, Wagle SR, Kovacevic B, et al. Bile acid bio-nanoencapsulation improved drug targeted-delivery and pharmacological effects via cellular flux: 6-months diabetes preclinical study. Sci Rep. 2020;10(1):1-15.

58. Dyrszka H, Salen G, Zaki FG, Chen T, Mosbach E. Hepatic toxicity in the rhesus monkey treated with chenodeoxycholic acid for 6 months: biochemical and ultrastructural studies. Gastroenterology. 1976;70(1):93-104. doi:10.1016/S0016-5085(76)80409-X

59. Renga B, Mencarelli A, Vavassori P, Brancaleone V, Fiorucci S. The bile acid sensor FXR regulates insulin transcription and secretion. Biochim Biophys Acta Mol Basis Dis. 2010;1802(3):363-372. doi:10.1016/j.bbadis.2010.01.002

60. Mooranian A, Zamani N, Ionescu CM, et al. Oral gavage of nano-encapsulated conjugated acrylic acid-bile acid formulation in type 1 diabetes altered pharmacological profile of bile acids, and improved glycaemia and suppressed inflammation. Pharmacol Rep. 2020;72:1-11. 
61. Gong Z, Zhou J, Zhao S, et al. Chenodeoxycholic acid activates NLRP3 inflammasome and contributes to cholestatic liver fibrosis. Oncotarget. 2016;7(51):83951. doi:10.18632/oncotarget.13796

62. Xie K, Xu B, Zhang Y, et al. A multi-method evaluation of the effects of Inflammatory cytokines (IL- $1 \beta$, IFN- $\gamma$, TNF- $\alpha$ ) on pancreatic $\beta$-cells. J Cell Physiol. 2018;233(12):9375-9382. doi:10.1002/jcp.26518

63. Oshi MA, Naeem M, Bae J, et al. Colon-targeted dexamethasone microcrystals with $\mathrm{pH}$-sensitive chitosan/alginate/Eudragit $\mathrm{S}$ multilayers for the treatment of inflammatory bowel disease. Carbohydr Polym. 2018;198:434 442. doi:10.1016/j.carbpol.2018.06.107

64. Barthson J, Germano CM, Moore F, et al. Cytokines tumor necrosis factor- $\alpha$ and interferon- $\gamma$ induce pancreatic $\beta$-cell apoptosis through STAT1-mediated Bim protein activation. J Biol Chem. 2011;286 (45):39632-39643. doi:10.1074/jbc.M111.253591

65. Ward JB, Lajczak NK, Kelly OB, et al. Ursodeoxycholic acid and lithocholic acid exert anti-inflammatory actions in the colon. $\mathrm{Am}$ J Physiol Gastrointest Liver Physiol. 2017;312(6):G550-G558. doi:10.1152/ajpgi.00256.2016

66. Horikawa T, Oshima T, Li M, et al. Chenodeoxycholic acid releases proinflammatory cytokines from small intestinal epithelial cells through the farnesoid X receptor. Digestion. 2019;100(4):286-294. doi: $10.1159 / 000496687$
67. Mooranian A, Ionescu CM, Wagle SR, et al. Probucol Pharmacological and Bio-Nanotechnological Effects on Surgically Transplanted Graft Due to Powerful Anti-Inflammatory, Anti-Fibrotic and Potential Bile Acid Modulatory Actions. Pharmaceutics. 2021;13(8):1304. doi:10.3390/pharmaceutics13081304

68. Wagle SR, Kovacevic B, Ionescu CM, et al. Pharmacological and Biological Study of Microencapsulated Probucol-Secondary Bile Acid in a Diseased Mouse Model. Pharmaceutics. 2021;13(8):1223. doi:10.3390/pharmaceutics 13081223

69. Mooranian A, Foster T, Ionescu CM, et al. Enhanced Bilosomal Properties Resulted in Optimum Pharmacological Effects by Increased Acidification Pathways. Pharmaceutics. 2021;13(8):1184. doi:10.3390/pharmaceutics13081184

70. Mooranian A, Jones M, Ionescu CM, et al. Advancements in Assessments of Bio-Tissue Engineering and Viable Cell Delivery Matrices Using Bile Acid-Based Pharmacological Biotechnologies. Nanomaterials. 2021;11(7):1861. doi:10.3390/ nano11071861

\section{Publish your work in this journal}

Drug Design, Development and Therapy is an international, peerreviewed open-access journal that spans the spectrum of drug design and development through to clinical applications. Clinical outcomes, patient safety, and programs for the development and effective, safe, and sustained use of medicines are a feature of the journal, which has also been accepted for indexing on PubMed Central. The manuscript management system is completely online and includes a very quick and fair peer-review system, which is all easy to use. Visit http://www. dovepress.com/testimonials.php to read real quotes from published authors. 